\title{
Reversible Hemianopsia Caused by Mechanical Compression of the Visual Pathway by the Dilated Draining Vein of an Arteriovenous Malformation
}

\section{-Case Report-}

\author{
Tomoji TAKIGAWA, Yuji MATSUMARU, Mikito HAYAKAWA, \\ Noriaki FUKUHARA*, Masaaki Usui*, and Akira MATSUMURA** \\ Departments of Endovascular Neurosurgery and *Neurosurgery, Toranomon Hospital, Tokyo; \\ **Department of Neurosurgery, Institute of Clinical Medicine, Graduate School of \\ Comprehensive Human Sciences, University of Tsukuba, Tsukuba, Ibaraki
}

\begin{abstract}
A 41-year-old woman presented with progressive hemianopsia caused by compression of the lateral geniculate body by the dilated basal vein draining a contralateral frontal arteriovenous malformation (AVM). Magnetic resonance (MR) imaging revealed left frontal AVM and right lateral geniculate body compression due to the dilated basal vein. Emergent presurgical transarterial embolization and surgical removal were performed. Left hemianopsia completely recovered 3 months after surgery and MR imaging indicated improvement of the compression of the lateral geniculate body. Direct mechanical compression of the enlarged drainage vein is one of the causes of homonymous hemianopsia. Early surgical treatment is recommended to obtain a rapid recovery.
\end{abstract}

Key words: arteriovenous malformation, dilated drainage vein, direct mechanical compression, lateral geniculate body, reversible hemianopsia

Received September 24, 2009; Accepted November 26, 2009

Author's present address: T. Takigawa, M.D., Department of Neurosurgery, Institute of Clinical Medicine, Graduate School of Comprehensive Human Sciences, University of Tsukuba, Tsukuba, Ibaraki, Japan. 


\section{Introduction}

Arteriovenous malformations (AVMs) are believed to arise from congenital maldevelopment of intracranial vessels. Embryological shunts persist after the failure of capillaries to develop between arteries and veins. Intracranial AVMs may manifest as subarachnoid hemorrhages, seizures, headaches, bruits, and focal neurological deficits that are sometimes progressive. ${ }^{12,13)}$ Homonymous hemianopsia on the contralateral side is the most common neuroophthalmological sign of intracranial AVMs. ${ }^{3,4)}$ Intracranial hemorrhage, arterial steal phenomena, and venous ischemia have been described as the cause of the symptoms, especially with AVMs in the occipital lobe. However, neurological anatomy would predict homonymous hemianopsia on the ipsilateral side of the AVM.

We treated a 41-year-old woman with a left frontal AVM manifesting as left homonymous hemianopsia whose clinical course and angioarchitecture strongly implicated compression of the lateral geniculate body by dilated venous drainage as the cause of the visual field deficit.

\section{Case Report}

A 41-year-old woman suffered an episode of generalized seizure. Magnetic resonance (MR) imaging revealed left frontal AVM and dilation of the left cortical vein (Fig. 1). Carotid angiography demonstrated an AVM in the left frontal region supplied by the left anterior and middle cerebral arteries, and mainly draining into the superior sagittal sinus (SSS) through the left cortical veins (Fig. 2). Surgical removal was recommended, but the patient preferred to opt for a more conservative treatment.

Six years later, she was admitted to our hospital for visual field defects of the left temporal side. Ophthalmological examination revealed normal visual acuity and the Goldmann perimeter found superior quadrantic hemianopsia in both eyes (Fig. 3) without optic atrophy. MR imaging showed no enlargement of the AVM nidus, but demonstrated apparent dilation of the cortical veins in both hemispheres. Furthermore, the right lateral genicu-

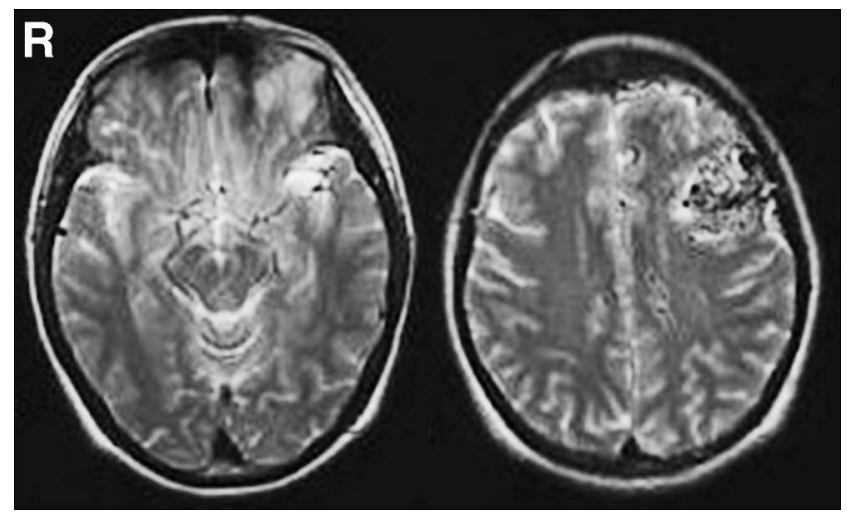

Fig. $1 T_{2}$-weighted magnetic resonance images obtained 6 years before admission showing the left frontal arteriovenous malformation. late body was compressed by the dilated basal vein with perifocal edema, not detected by the previous MR imaging (Fig. 4). Cerebral angiography revealed marked changes of the venous drainage pattern due to the occlusion of the SSS. Antegrade drainage through the SSS was obstructed, so retrograde drainage through right cortical veins predominated from the left cortical veins. As a result, the right basal vein was enlarged (Fig. 5). On the next day, she noticed aggravation of the visual field deficit, and the Gol-

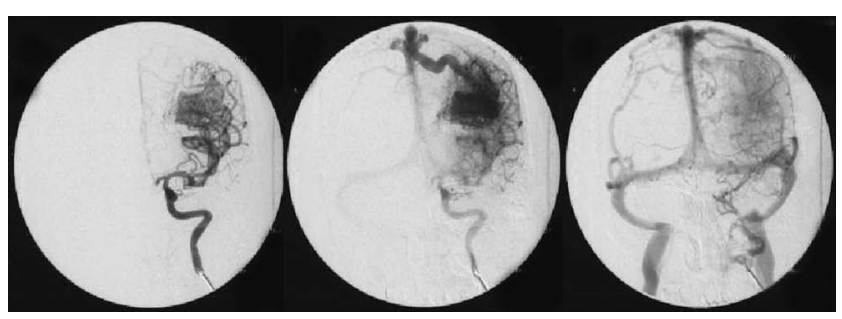

Fig. 2 Left internal carotid angiograms obtained 6 years before admission demonstrating the left arteriovenous malformation fed by the left anterior and middle cerebral arteries, and mainly drained by the left superficial vein to the superior sagittal sinus.

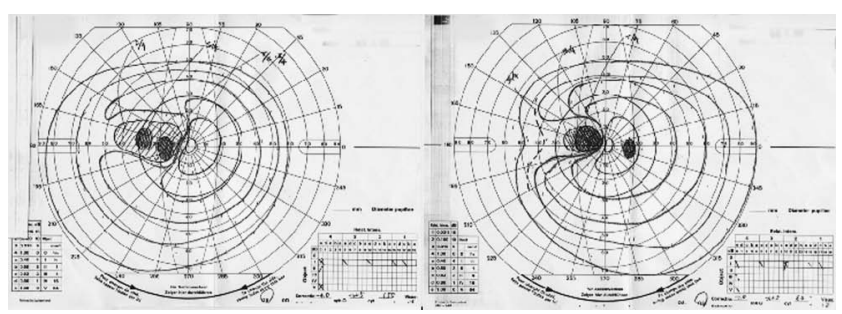

Fig. 3 Goldmann perimeter of the visual field showing left incomplete quadrantic hemianopsia at the time of hospitalization.
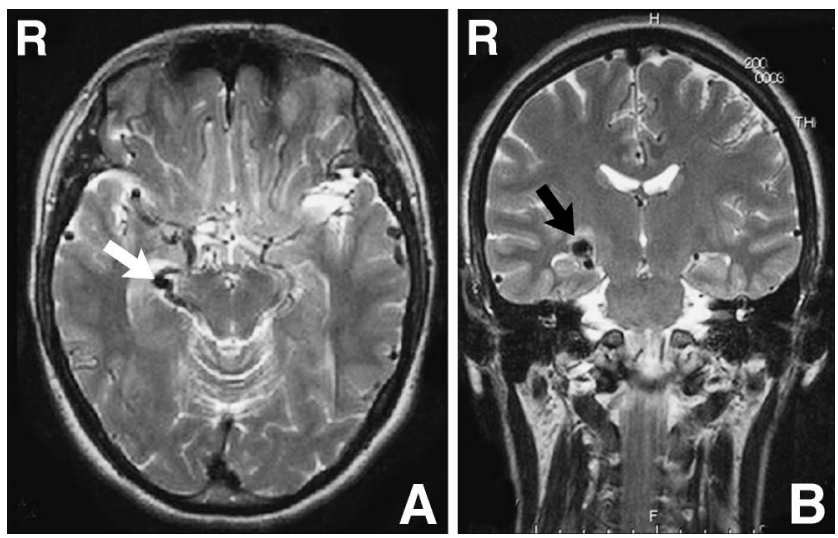

Fig. 4 A: Axial $T_{2}$-weighted magnetic resonance image showing enlargement of the right basal vein and compression of the right lateral geniculate body (arrow). B: Coronal $\mathrm{T}_{2}$-weighted magnetic resonance image showing compression of the right lateral geniculate body with perifocal edema (arrow). 


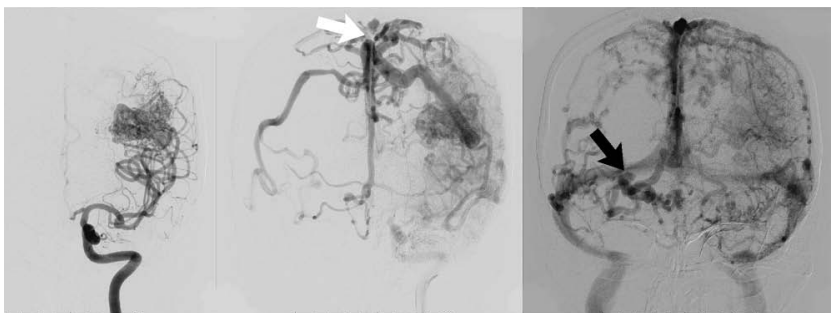

Fig. 5 Left internal carotid angiograms revealing occlusion of the superior sagittal sinus (white arrow), and venous drainage via the enlarged right basal vein (black arrow) through the right cortical vein.

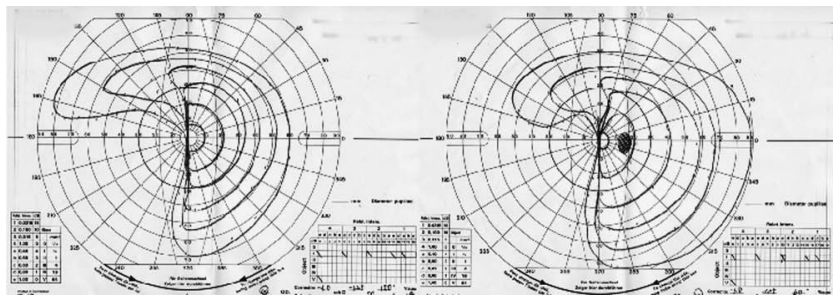

Fig. 6 Goldmann perimeter of the visual field showing expansion of the left hemianopsia on the day after angiography.

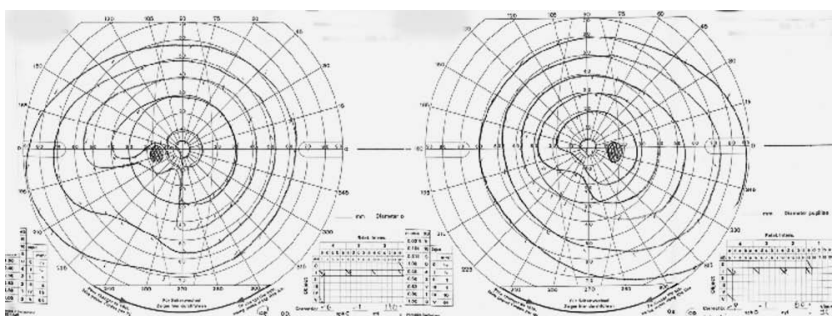

Fig. 7 Goldmann perimeter of the visual field showing improvement of the left hemianopsia 3 months after arteriovenous malformation removal.

dmann perimeter showed expansion of the visual field deficits in both eyes (Fig. 6). MR imaging showed no evidence of hemorrhage or infarction. We speculated that the compression of the contralateral lateral geniculate body or the optic tract by the dilated basal vein had caused aggravation of the visual field deficit. Presurgical transarterial embolization was successfully completed without complications.

The surgical outcome was favorable, and clinical improvement was observed immediately after the operation. One week after surgery, her left hemianopsia had improved to the preoperative state at the time of hospitalization. Three months after surgery, her visual field had completely recovered, except for reduced sensitivity in the lower left quadrant of the left eye (Fig. 7). MR imaging showed improvement in the compression of the right lateral geniculate body and perifocal edema (Fig. 8).

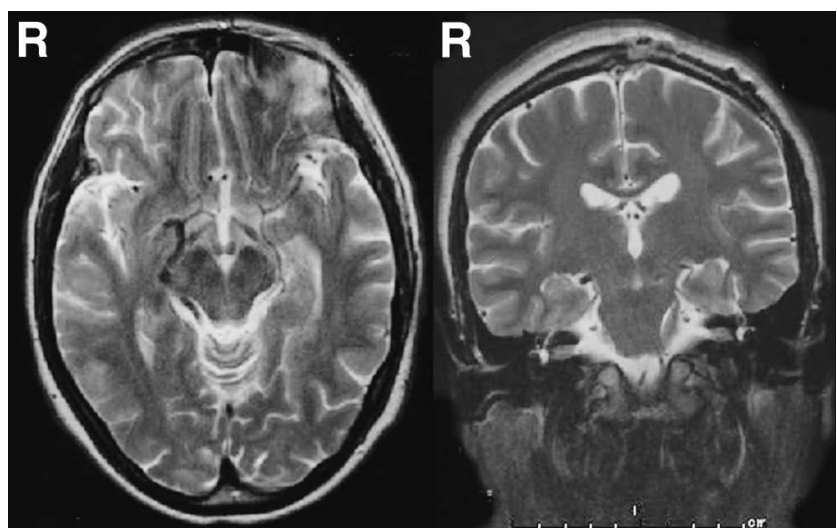

Fig. $8 T_{2}$-weighted magnetic resonance images obtained 3 months after the surgery showing improvement in the dilated basal vein, compression of the lateral geniculate body, and perifocal edema.

\section{Discussion}

Discovering hemianopsia in a relatively young patient is alarming, and raises ominous prospects, particularly for tumors or, rarely, demyelination. ${ }^{5)}$ Unilateral occipital lesions in older patients are most commonly due to stroke. Tumors in the occipital regions include meningiomas, gliomas, and metastatic lesions. Trauma or recent manipulation can be relevant. Complicated migraine can cause permanent hemianopsia. ${ }^{10,15)}$ Hemianopsia due to AVM is relatively rare, so would not be readily considered in the differential diagnosis.

Multiple mechanisms have been proposed to explain hemianopsia caused by AVMs in the central nervous system, including hemorrhages, arterial steal phenomena, and diverse mechanisms resulting from interference with venous drainage such as thrombosis, increased intracranial pressure, venous ischemia, and hydrocephalus. Contralateral homonymous visual field deficit is the most common neurological deficit, occurring in $67-90 \%$ of patients with occipital AVMs that have hemorrhaged.1,8,11,14] Venous ischemia often causes neurological deficit in patients with tentorial dural AVMs associated with visual hallucinations and quadrantic hemianopsia.6,9) One case of tentorial dural arteriovenous fistula presenting with superior quadrantic hemianopsia was due to dilated venous drainage compressing the visual pathway. ${ }^{2)}$ Three AVM cases with hemianopsia and optic nerve atrophy were due to compression of the lateral geniculate body by enlarged venous drainage. ${ }^{7)}$ Mechanical compression of the optic pathway by dilated drainage vein is clearly a potential cause.

In our case with left frontal AVM, the deficit was in the right visual pathway. The SSS was occluded and the right basal vein served as the drainage pathway and consequently was dilated. Single photon emission computed tomography revealed no laterality and MR imaging depicted the dilated right basal vein and perifocal edema of the right lateral geniculate body, so we concluded that the symptom was due to the dilated drainage vein on the other 
side of the AVM, which in turn compressed the lateral geniculate body without venous congestion. Such cerebral AVM manifesting as homonymous hemianopsia of the ipsilateral side is extremely rare.

Visual field deficit worsened or was unchanged after surgical intervention in $44-70 \%$ of patients with occipital AVM. ${ }^{1,8,11,14)}$ Visual field deficit caused by dilated vein was also irreversible in cases with optic atrophy caused by long-standing compression, ${ }^{7)}$ but is reversible in patients without ophthalmoscopic abnormalities. Immediate treatment is essential for these patients to cure the visual deficit.

The present rare case of homonymous hemianopsia associated with AVM was caused by direct mechanical compression of the contralateral dilated drainage vein. Several mechanisms have been proposed to explain the symptoms caused by AVM, but direct compression of the drainage vein is one of the causes of homonymous hemianopsia. The condition may be reversible if treated at an early stage.

\section{References}

1) Bartolomei J, Wecht DA, Chaloupka J, Fayad P, Awad IA: Occipital lobe vascular malformations: prevalence of visual field deficits and prognosis after therapeutic intervention. Neurosurgery 43: 415-421, 1998

2) Benndorf G, Schmidt S, Sollmann WP, Kroppenstedt SN: Tentorial dural arteriovenous fistula presenting with various visual symptoms related to anterior and posterior visual pathway dysfunction: case report. Neurosurgery 53: 222226, 2003

3) Dimsdale H, Hobbs E: Discussion on the neuro-ophthalmological aspects of the cerebral angiograms. Proc R Soc Med 50: 85-92, 1957

4) Enoksson P, Bynke H: Visual field defects in arteriovenous aneurysms of the brain. Acta Ophthalmol (Copenh) 36: 586-600, 1958

5) Gutteridge IF: An unusual ocular presentation of multiple sclerosis. Am J Optom Physiol Opt 62: 69-71, 1985

6) Inafuku T, Sakai F, Sakamoto T, Iizuka T, Kanda T: [Visual hallucination in the hemianopic field caused by dural ar- teriovenous malformation]. Rinsho Shinkeigaku 34: 484-488, 1994 (Jpn)

7) Kupersmith MJ, Vargas M, Hoyt WF, Berenstein A: Optic tract atrophy with cerebral arteriovenous malformations: direct and transsynaptic degeneration. Neurology 44: 80-83, 1994

8) Kupersmith MJ, Vargas ME, Yashar A, Madrid M, Nelson K, Seton A, Berenstein A: Occipital arteriovenous malformations: visual disturbances and presentation. Neurology 46: 953-957, 1996

9) Kurata A, Miyasaka Y, Yoshida T, Kunii M, Yada K, Kan S: Venous ischemia caused by dural arteriovenous malformation. Case report. J Neurosurg 80: 552-555, 1994

10) Lewis RA, Vijayan N, Watson C, Keltner J, Johnson CA: Visual field loss in migraine. Ophthalmology 96: 321-326, 1989

11) Martin NA, Wilson CB: Medial occipital arteriovenous malformations. Surgical treatment. J Neurosurg 56: 798-802, 1982

12) Perret G, Nishioka H: Report on the cooperative study of intracranial aneurysms and subarachnoid hemorrhage. Section VI. Arteriovenous malformations. An analysis of 545 cases of cranio-cerebral arteriovenous malformations and fistulae reported to the cooperative study. J Neurosurg 25: 467-490, 1966

13) Stein BM, Wolpert SM: Arteriovenous malformations of the brain. I: Current concepts and treatment. Arch Neurol 37: $1-5,1980$

14) Troost BT, Newton TH: Occipital lobe arteriovenous malformations. Clinical and radiologic features in 26 cases with comments on differentiation from migraine. Arch Ophthalmol 93: 250-256, 1975

15) Wakakura M, Ichibe Y: Permanent homonymous hemianopias following migraine. J Clin Neuroophthalmol 12: 198202, 1992

Address reprint requests to: Tomoji Takigawa, M.D., Department of Neurosurgery, Institute of Clinical Medicine, Graduate School of Comprehensive Human Sciences, University of Tsukuba, 1-1-1 Tennodai, Tsukuba, Ibaraki 305-8575, Japan.

e-mail: t-tomojy@mx2.harmonix.ne.jp 